\title{
Constant pressure air charging cascade control system based on fuzzy PID controller
}

\author{
Jun Zhao \& Zheng Zhang \\ College of information science and engineering, Wuhan University of science and technology, \\ Wuhan 430081, China
}

\begin{abstract}
Keywords: Fuzzy PID control; Constant pressure air charging; Pneumatic gas booster; Cascade control.
\end{abstract}

\begin{abstract}
Aiming at the problem of air charging safe, high efficient and energy-saving with pneumatic gas booster ,a constant pressure air charging system was designed to rapid filling respirator ,and the fire respirator`s com-pressed air bottle fast filling station was transformed into air charging experiment platform, the controller combined the cascade control, principle of fuzzy control and conventional PID control algorithm ,and realized the Real-time adjustment of the PID parameters, by automatically adjusting the pressure of pneumatic gas booster`s drive circuit to realize automatic air charging in a constant pressure. The simulation and experiment results show that the system has a very good dynamic, static performance and the actual control effect, it pro-vided a realization method of constant pressure air charging gas booster.
\end{abstract}

\section{Introduction}

Fire respirator`s compressed air bottle fast filling station is an inflation equipment for air respirator. It is widely used in petroleum, chemical, marine, fire, smelting, factories and mines, etc. In long time fire operations, firefighters who entered the anoxic, toxic, harmful gases and etc. fire rescue scene, need to consume large amounts of compressed breathing air supply. Restricted by the max number of air respirators that can be configured, it is required that the fire respirator`s compressed air bottle fast filling station can inflate the respirators under a safe pressure value fast, safe, clean and efficiently, thus ensues the smooth progress of the fire-fighting task. At present, the design and application of constant pressure inflation system has attracted widespread attention.

In general, classified by the types of gas supercharging equipment, there are two kinds of inflation systems: one is the constant pressure inflation systems based on electric-drive gas supercharging equipment. Zhu Yinghuang, Yao Shikuan et al. renovated the traditional compressor by constant pressure variable frequency technique using frequency converter with conventional PID control method, which extends the life span of equipment, achieves energy saving, and ensures the stability of the pipe network. Wang Jinghui et al. designed a constant pressure gas supplying compressor system based on fuzzy control, which improves the reliability and stability of the system. Bai Kunhai, Qu Xusheng et al. who designed the fuzzy-PID control system by combining the principle of the fuzzy control and conventional PID control and verify its superiority. Cai Kailong et al. proposed an adaptive PID controller based on neural network which controls the pressure of constant pressure gas supplying system by stepper motor. The other is the constant pressure inflation system based on gas-drive supercharging equipment, which is used in places with a high-pressure gas source, such as large ships. Compared with system using electric-drive supercharging equipment, system using gas-drive supercharging equipment does not produce heat, sparks, need no external lubricants, can start and stop continuously, which is light, reliable and easy to maintain. Faced with constant pressure inflation system using gas-drive supercharging equipment, the algorithm above has certain limitations. With the increasingly use of inflatable devices based on gas-drive supercharging equipment, it has a very important application value to design safe and energy-saving constant pressure automatic inflation system.

This paper studies on compressed air fast inflating station for cylinders of fire-fighting respirators based on gas-drive supercharging equipment which applied to large ships. According to the gas-drive 
gas booster pump's nonlinear, large time delay characteristics, the inflatable constant pressure cascade control system based on fuzzy PID's is designed. This system realizes constant-pressure, stable, safe, fast inflation of a plurality of high-pressure gas cylinders by adjusting the driving pressure of the gas booster pump automatically. Fire respirator`s compressed air bottle fast filling station was transformed into air charging experiment platform, which is used to verify the effectiveness and superiority of the fuzzy PID cascade control algorithm.

\section{Design OF Constant pressure air charging cascade control system}

\subsection{Control program design of constant pressure air charging system}

Constant pressure air charging system is a system of inflation pressure control, this system is required to filling 1-4 6.8L respirator as soon as possible, and at the same time, to prevent the gas bottle mouth be filled with explosive hazards, etc. It`s main objective is control the inflate pressure under a safe value (30Mpa) after gas booster started, and automatically stop charging when the cylinder respirator`s pressure increased to 30Mpa. According to the technological requirements, design of Schematic diagram of the control system as shown in figure 1:

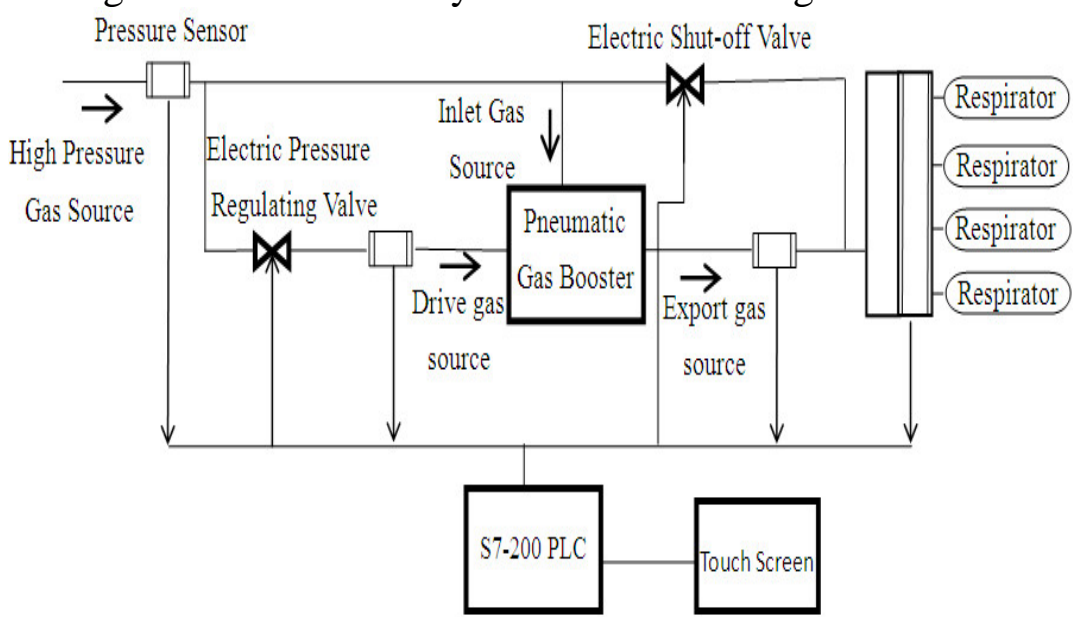

Figure 1.Schematic diagram of the control system.

\subsection{Air charging process design}

In order to achieve high efficiency and energy saving, the whole process is divided into two stage, natural air charging stage and booster air charging stage.

The first stage is natural air charging stage. Make full use of high pressure gas source(about $10 \mathrm{Mpa}$ ), it`s much larger than the respirator`s pressure(approximately 6Mpa), this stage is not enabled pneumatic gas booster, just open electric shut-off valve and direct inflatable via Inflatable loop bypass. At the same time, as the air pressure between respirator and high pressure gas source get smaller, inflatable process get slower, when the pressure difference reaches a given pressure value, end natural air charging stage.

The second stage is booster air charging stage. After natural air charging stage, constant pressure air charging system automatically shut down the electric shut-off valve, and turn on electric pressure regulator valve, by adjusting the Drive gas source pressure to achieve constant pressure inflate.

\subsection{Cascade Control System Design}

At the booster air charging stage, constant pressure air charging system maintained the inflate pressure at a safe value (30Mpa) through adjusting the drive air pressure. If use the exports (inflate) pressure as the main signal back to the controller, the controller directly controls the electric pressure regulating valve, this single-loop control system shows its poor control performance, it is difficult to obtain better control quality. The reason is that the electric valve and the gas booster have a long distance and the driving pressure need time to regulate. Export (inflate) pressure lag and large inertia, long time delay, could easily lead to control is not timely, the overshoot increases and stability. Therefore, in order to improve the system response speed, improve the control quality, use the driving 
pressure as the auxiliary control signals, and design pressure-pressure air charging cascade control system, structure of the cascade control system as shown in Figure 2:

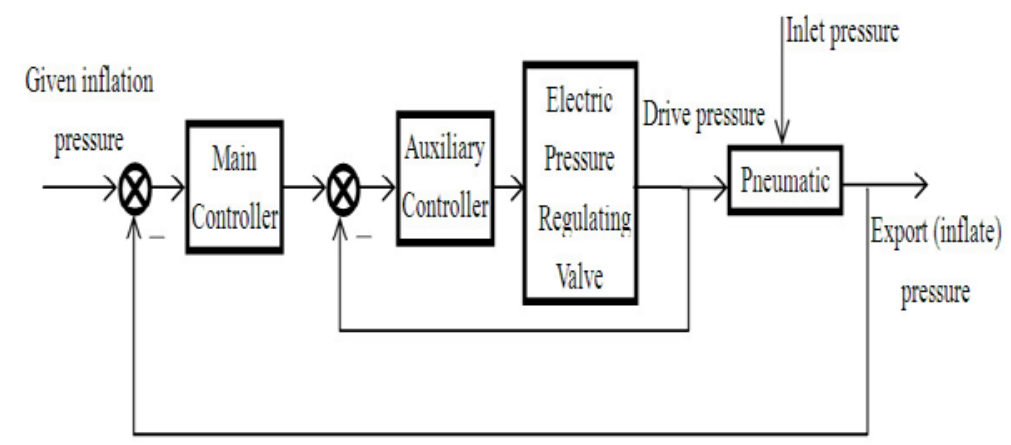

Figure 2.Structure of the cascade control system.

The purpose of the master controller is to control the gas booster pump outlet (inflatable) pressure. When there is a deviation of the inflation pressure and the set value of the actual inflation pressure detection, automatic control algorithm on the main controller calculates a control output, the control output that is a given sub-controllers. Practice and experience shows that the constant pressure inflation system is a complex system of nonlinear, large delay. Conventional PID controller tuning parameters are often poor, poor performance, poor adaptability of the controlled process; fuzzy controller is not required to master the mathematical model of controlled object, but it is generally not high control accuracy. So the master controller using fuzzy control and PID control methods combine the two, weaknesses, both fuzzy control and flexible, adaptable PID control also has the advantages of high accuracy.

Secondary loop is a servo system, it's a given value varies with changes in the master controller output. In order to drive the output pressure of the master controller to quickly follow and remain stable given, deputy controller preferably without integral role because the integral action will slow track; the derivative action is not needed, as deputy controller When the derivative action, once the master controller and a slight change in the output of the electric valve will change dramatically, and this control is unfavorable. In this system, the sub-controllers just using $\mathrm{P}$ control algorithm can achieve a good result.

\section{Fuzzy PID controller design}

\subsection{Fuzzy PID control theory}

Fuzzy PID controller is mainly composed by two parameters, PID controller and fuzzy inference section. Fuzzy controller select pressure error e and pressure error change rate $\Delta \mathrm{e}$ as input; and three parameters proportion: $\Delta \mathrm{kP}$, integration: $\Delta \mathrm{ki}$, differential: $\Delta \mathrm{kd}$ as output. Through fuzzy inference to adjust the parameters KP, KI, KD online as the e and $\Delta \mathrm{e}$ changed. So that the controlled object has a good dynamic and static performance. Fuzzy PID controller structure as show in Figure 3:

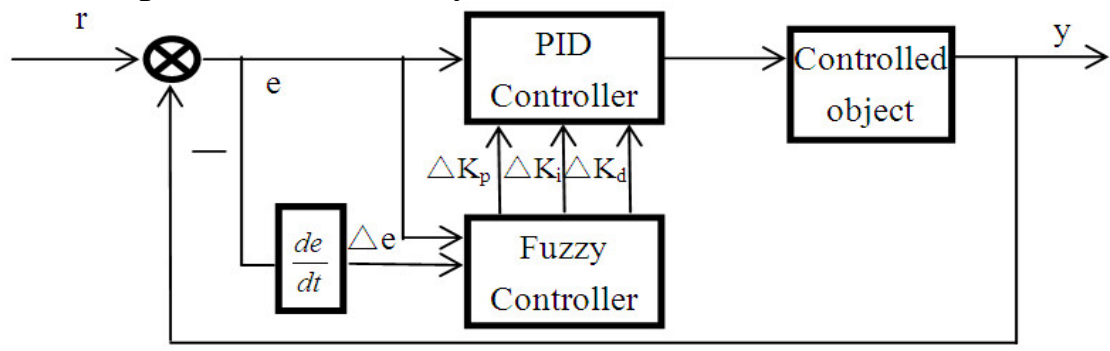

Figure 3.Structure of the fuzzy PID controller.

\subsection{Fuzzy sets and membership}

Fuzzy controller input, output basic discourse domain:

e discourse domain $[-1,1]$; 
$\Delta \mathrm{e}$ discourse domain $[-0.1,0.1]$

$\triangle \mathrm{kP}$ discourse domain $[-0.2,0.2]$;

$\Delta \mathrm{ki}$ discourse domain [-0.05,0.05];

$\Delta \mathrm{kd}$ discourse domain [-0.02,0.02]。

The fuzzy domain unified as $[-6,6]$. Fuzzy set is unified to $\{\mathrm{NB}, \mathrm{NM}, \mathrm{NS}, \mathrm{Z}, \mathrm{PS}, \mathrm{PM}, \mathrm{PB}\}$ were negative, negative, negative, zero, is small, middle, large.

Select the trigonometric function as membership function of $\mathrm{E}$ and $\Delta \mathrm{E}$.

$$
u(x)=\left\{\begin{array}{l}
\frac{x-a}{b-a}, x \in(a, b) \\
\frac{x-c}{b-c}, x \in(a, b)
\end{array}\right.
$$

The parameters of a and $b$ values from -6 to 6 .

Select normal distribution function as membership function of $\Delta \mathrm{kP}, \Delta \mathrm{ki}$ and $\Delta \mathrm{kd}$.

$$
u(x)=e^{-\frac{\left(x-x_{0}\right)^{2}}{2 \delta^{2}}} \text {, The } \delta \text { is the standard deviation. }
$$

\subsection{Fuzzy control rules}

According to the different fuzzy quantization value of e and $\Delta \mathrm{e}$, analysis and summary of control rules are as follows:

(1) When $|E|$ have large values, large selection of $\Delta K p$ to speed up the system response, in order to prevent the super harmonic and differential overflow, select a small $\Delta \mathrm{Ki}$ and $\Delta \mathrm{Kd}$ is zero.

(2)When $|E|$ have medium size values, in order makes the system has a small super harmonic, choose a small $\Delta \mathrm{Kp}$, a moderate $\Delta \mathrm{Ki}$ can guarantee the response speed, $\Delta \mathrm{Kd}$ have a greater impact on the system, so select a small $\Delta \mathrm{Kd}$.

(3) When $|E|$ is relatively small, in order to ensure the good steady state performance of the system, select the larger $\Delta \mathrm{Ki}$ and larger $\Delta \mathrm{Kp}$. At the same time, in order to avoid system oscillation near the set point and improve the anti-interference ability of the system, when $|\Delta \mathrm{E}|$ have large values, select smaller $\Delta \mathrm{Kd}$; when $|\Delta \mathrm{E}|$ have small values, select larger $\Delta \mathrm{Kd}$.

\subsection{Defuzzification}

Defuzzification using method of the weighted average method, the calculation formula is as follows:

$$
y=\frac{\sum_{1}^{m} x_{i} u\left(x_{i}\right)}{\sum_{1}^{m} u\left(x_{i}\right)},
$$

$X_{i}$ represents an element in the domain of fuzzy theory, $\mathrm{u}\left(\mathrm{X}_{\mathrm{i}}\right)$ represents its membership degree, value of $m$ represents fuzzy membership is not the number 0 .

Get the value of $\Delta \mathrm{Kp}, \Delta \mathrm{Ki}$ and $\Delta \mathrm{Kd}$ through the weighted average method. The output of fuzzy controller:

$$
\begin{aligned}
& \Delta k_{p}=K_{k_{p}} \times \Delta K_{P}, \Delta k_{i}=K_{k_{i}} \times \Delta K_{i}, \\
& \Delta k_{d}=K_{k_{d}} \times \Delta K_{d},
\end{aligned}
$$

Among them, ${ }^{K}{ }_{k p},{ }^{K}{ }_{k_{i}}$ and $K_{k_{d}}$ is quantification factor of $\Delta \mathrm{kP}, \Delta \mathrm{ki}$ and $\Delta \mathrm{kd}$.

$$
\begin{aligned}
& K_{k_{p}}=\frac{0.2}{6}=0.0333, K_{k_{i}}=\frac{0.05}{6}=0.0083, \\
& K_{k_{d}}=\frac{0.02}{6}=0.0033 .
\end{aligned}
$$

So the final parameter values for the PID control:

$$
k_{p}=k_{p 0}+\Delta k_{P}, k_{i}=k_{i 0}+\Delta k_{i}, k_{d}=k_{d 0}+\Delta k_{d} \text {, }
$$


Among them, $\mathrm{k}_{\mathrm{p} 0}, \mathrm{k}_{\mathrm{i} 0}, \mathrm{~K}_{\mathrm{D} 0}$ as the PID controller by the original value, site commissioning experience.

\section{Simulation and experimental results}

Pneumatic gas booster start with the initial state, and then inflate the pipe network with constant pressure, the inflatable pressure stabilize after the two processes:

(1) Pneumatic gas booster gradually sent gas to the pipe network, and this is a lag in the process;

(2)Gas is gradually filled the pipe network, gas pressure gradually increased until stability which is a process of inertia.

So according to the practice and experience, can be said that the mathematical model of pneumatic gas booster and electric valve are equivalent to one order inertia and pure lag system, its transfer function: $G_{s}=\frac{K e^{-\tau s}}{T s+1}$.

After identification, the parameters of the mathematical model of gas booster pump: $K=21, T=38$, =5.7. Parameters of electric valve mathematical model: $\mathrm{K}=1.3, \mathrm{~T}=8,=2.6$. Set the initial parameters of PID: $\mathrm{kP}=0.5, \mathrm{ki}=0.002, \mathrm{kd}=0.8$. The constant pressure air charging cascade control system simulation model establish by using Simulink and fuzzy control box in MATLAB, as shown in figure 4.

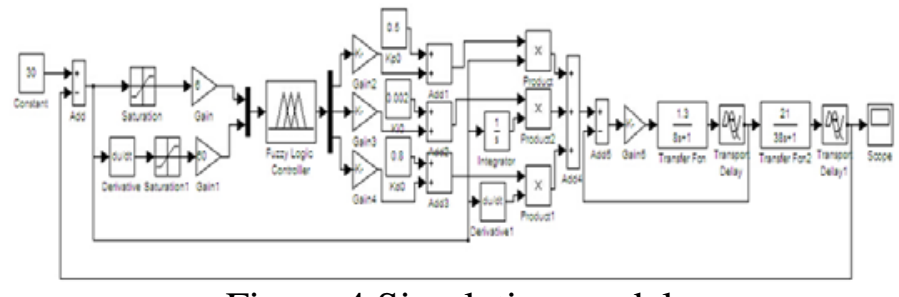

Figure 4.Simulation model

System simulation of the response curve as shown in Figure 5, the curves of the 1 output for cascade control based on fuzzy PID, curve of 2 output for single loop control system fuzzy PID control, curves 3 of output for traditional control system based on PID.

As can be seen from Figure 5, compared with the traditional PID control, fuzzy PID control has faster response, produced only minimal overshoot, faster at steady state. The cascade control based on fuzzy PID relative to the fuzzy PID based on single loop control has faster response, better control effect.

The actual inflation experiment results are shown in Table 1, we can see that the constant pressure air charging cascade control system based on fuzzy PID controller can inflatable 4 6.8L respirators in 5 minutes, and the charging time relative to the fuzzy PID control is reduced by $13 \%$, can be very good to meet the actual demand of filling respirator $\mathrm{s}$.

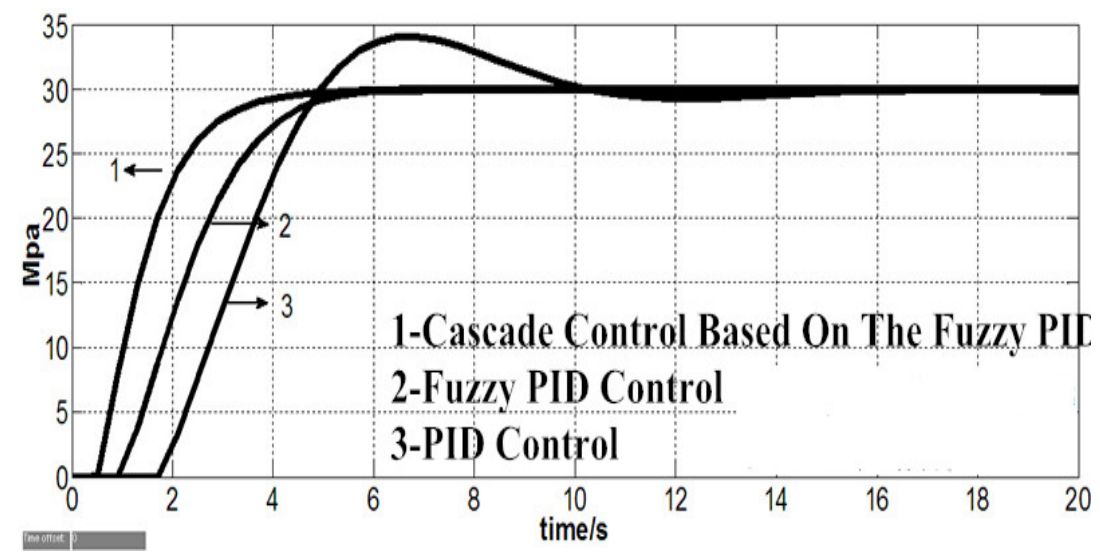

Figure 5.The simulation results 
Table 1.The result of air charging experiment

Number of respirator Cascade control based on fuzzy PID fuzzy PID

\begin{tabular}{lll}
\hline 1 & $1.259 \mathrm{~min}$ & $1.508 \mathrm{~min}$ \\
2 & $2.617 \mathrm{~min}$ & $3.015 \mathrm{~min}$ \\
4 & $5.235 \mathrm{~min}$ & $6.030 \mathrm{~min}$ \\
\hline
\end{tabular}

\section{Summary}

Point at the fire respirator`s compressed air bottle fast filling station based on pneumatic gas booster, designed a constant pressure air charging cascade control system based on fuzzy PID controller, through full use of the high pressure gas source, the natural air charging stage achieved a good energy saving effect. The simulation results show that, the method can play the advantages of fuzzy control, PID control and cascade control. The system can be stabilized in a shorter period of time, smaller overshoot, and effectively eliminating adverse effects of large delay, large inertia and high pressure gas source fluctuation to system during the charging process, and achieved the purpose of security, efficient.

\section{References}

[1] Bai, Kunhai. 1974. Design of constant pressure supply air system based on fuzzy-PID. Chinese Hydraulics \& Pneumatics 2012, (6):53-55.

[2] Cai, Kailong. 1979. Application of neural network PID control in constant pressure air feeding system based on step motor. Chinese Hydraulics \& Pneumatics 2006, (6):63-66.

[3] Wang, Jinhui. 1978. Fuzzy control-based constant pressure centroI for air compressors. Control and Instruments in Chemical Industry 2011, 38(6):689-690.

[4] Zhu, Yinghuang. 1967. Application of frequency inverter in constant pressure control of air compressor. Process Automation Instrumentation 2009, 30(1):66-69.

[5] Zhai, Shikuan. 1979. Inverter in transformation of air compressorconstant pressure air supply. Electric Machines \& Control Application 2012, 39(3):40-43.

[6] Zhai, Xusheng. 1984. A constant pressure feeding system based on adaptive fuzzy-PID control. Chinese Hydraulics \& Pneumatics 2008, (2):21-23. 